\title{
The Extracellular Matrix Modulates Olfactory Neurite Outgrowth on Ensheathing Cells
}

\author{
Katarina T. Tisay ${ }^{1}$ and Brian Key ${ }^{2}$ \\ ${ }^{1}$ Department of Anatomy and Cell Biology, University of Melbourne, Victoria 3052, Australia, and ${ }^{2}$ Neurodevelopment \\ Laboratory, Department of Anatomical Sciences, University of Queensland, Brisbane 4072, Australia
}

Primary olfactory axons grow along a stereotypical pathway from the nasal cavity to the olfactory bulb through an extracellular matrix rich in laminin and heparan sulfate proteoglycans (HSPGs) and bounded by the expression of chondroitin sulfate proteoglycans (CSPGs). This pathway is pioneered by olfactory ensheathing cells, which provide a substrate conducive for axon growth during early development. In the present study, we examined the effect of several extracellular matrix constituents on the spreading and migration, as well as the neurite outgrowth-promoting properties, of olfactory ensheathing cells. Laminin and Matrigel enhanced the spreading and migration of olfactory ensheathing cells and increased their neurite outgrowth-promoting activity. In contrast, HSPG and CSPG had little effect on the spreading and migration of olfactory ensheathing cells and hence did not promote olfactory neurite outgrowth. In vitro olfactory axons grew preferentially on the surface of olfactory ensheathing cells rather than the underlying extracellular matrix. We propose that olfactory ensheathing cells secrete laminin and HSPGs, which together with other cofactors, stimulate these cells to migrate and adopt a neurite outgrowth-promoting phenotype. Expression of CSPGs in the surrounding mesenchyme confines the growth of ensheathing cells, as well as the axons, which grow on the surface of these cells, to a specific pathway. Thus, the ECM indirectly modulates the growth and guidance of olfactory axons during development.

Key words: neurite outgrowth; laminin; proteoglycan; ensheathing cells; chondroitin sulfate; primary olfactory neuron
Olfactory ensheathing cells (OECs) are specialized glia that ensheathe bundles of primary olfactory axons. These cells are born in the olfactory neuroepithelium (OE) and migrate together with growing axons, populating both the olfactory nerve and nerve fiber layer of the olfactory bulb (Tennent and Chuah, 1996). OECs are a highly conducive substrate for primary olfactory axon outgrowth in vitro (Goodman et al., 1993; Chuah and Au, 1994; Key et al., 1996; Kafitz and Greer, 1998, 1999) and appear to be responsible for the unique ability of these axons to reinnervate the olfactory bulb during regeneration (RamónCueto and Valverde, 1995). When OECs are transplanted into damaged spinal cord, they also facilitate the regrowth of spinal axons through the lesion (Li et al., 1997, 1998; Ramón-Cueto et al., 1998).

OECs are not lineally related to Schwann cells in other peripheral nerves; they are instead derived from progenitor cells within the olfactory placode (Chuah and Au, 1991) and have phenotypic characteristics of both astrocytes and Schwann cells (Doucette, 1990). OECs lack a basal lamina and ensheathe large bundles of axons, much like astrocytes in the central tracts (DeLorenzo, 1957; Barber and Lindsay, 1982). These cells also contain low levels of central-type glial fibrillary acidic protein (GFAP), contribute to the glial limitans of the olfactory bulb, and form perivascular end feet on blood vessels, which has further likened them to astrocytes (Barber and Lindsay, 1982; Doucette, 1984,

Received June 11, 1999; revised Aug. 20, 1999; accepted Aug. 27, 1999.

This work was supported by National Health and Medical Research Council grants. K.T.T. was supported by an Australian Postgraduate Award and a Queen's Trust Young Achiever Award.

Correspondence should be addressed to Brian Key, Neurodevelopment Laboratory, Department of Anatomical Sciences, University of Queensland, Brisbane 4072, Australia. E-mail: brian.key@mailbox.uq.edu.au.

Copyright (C) 1999 Society for Neuroscience 0270-6474/99/199890-10\$05.00/0
1990). On the other hand, OECs like Schwann cells, express L1 (Ramón-Cueto and Nieto-Sampedro, 1992) and p75, the lowaffinity nerve growth factor receptor (Pixley, 1992), and they also myelinate dorsal root ganglion neurites in vitro (Devon and Doucette, 1992).

The spatiotemporal expression pattern of various components of the extracellular matrix in the developing olfactory nerve pathway suggests that these molecules may play an important role in the growth and guidance of primary olfactory axons. For instance, laminin, heparan sulfate proteoglycan (HSPG), and collagen IV are expressed along the olfactory nerve pathway (Doucette, 1996; Treloar et al., 1996; Whitesides and LaMantia, 1996). Moreover, these extracellular molecules promote the outgrowth of primary olfactory neurites in vitro (Whitesides and LaMantia, 1996; Kafitz and Greer, 1997). In contrast, chondroitin sulfate proteoglycans (CSPGs) are expressed in the mesenchyme along the boundary of the olfactory nerve and may possibly confine the growth of axons within the nerve fiber bundles, as has been proposed for dorsal root ganglion axons (Katoh-Semba et al., 1994).

Although the ECM may directly affect the growth of primary olfactory axons, we have suggested previously that it modulates the outgrowth-promoting properties of OECs (Key et al., 1996). In the present study, we have investigated the effect of various ECM components on the phenotypic characteristics of OECs, as well as their neurite outgrowth-promoting properties. We show that laminin and Matrigel, but not CSPG and HSPG, strongly promoted cell spreading and enhanced the neurite outgrowthpromoting activity of OECs.

\section{MATERIALS AND METHODS}

Explant cultures of olfactory neuroepithelium. Timed pregnant Sprague Dawley rats were anesthetized with Nembutal $(80 \mu \mathrm{l} / 100$ gm body 
weight) at embryonic day 19.5 (E19.5). The morning of positive sperm dam was designated E0.5. Embryos were removed aseptically and decapitated, and the posterior third of the nasal septum with attached olfactory neuroepithelium was removed. The olfactory neuroepithelium was peeled from the nasal septum and cut into explants $\sim 1 \mathrm{~mm}^{2}$ in size. Explants were plated onto $12 \mathrm{~mm}$ Millicell-M culture inserts (Millipore, Bedford, MA), which were coated previously with various substrates. Membranes were prepared by diluting each substrate in sterile PBS, $\mathrm{pH}$ 7.4, and incubating $100 \mu \mathrm{l}$ of each solution overnight at room temperature. Membranes were then washed three times with PBS and twice with warmed medium, before explant plating. The substrates used were as follows: poly-L-lysine (PLL) (100 $\mu \mathrm{g} / \mathrm{ml}$; Sigma, St Louis, MO), Matrigel basement membrane matrix secreted by Englebreth-Holm-Swarm (EHS) mouse sarcoma cells (1540 $\mu \mathrm{g} / \mathrm{ml}$; Collaborative Research, Bedford, MA), EHS-laminin (100 $\mu \mathrm{g} / \mathrm{ml}$; Collaborative Research), EHSHSPG (10 $\mu \mathrm{g} / \mathrm{ml}$; Sigma), and CSPG A from bovine trachea $(10 \mu \mathrm{g} / \mathrm{ml}$; Sigma). Cultures were maintained in DMEM (Sigma) containing 10\% fetal bovine serum (v/v), Monomed serum-free hybridoma supplement (Commonwealth Serum Laboratories, Melbourne, Australia), and gentamicin $(50 \mu \mathrm{g} / \mathrm{ml})$. In some experiments, cytosine arabinoside $(10 \mu \mathrm{M}$; Sigma) and 5-fluoro-2'-deoxyuridine (10 $\mu \mathrm{M}$; Sigma) were included in the culture medium. Explants were cultured for $5 \mathrm{~d}$ at $37^{\circ} \mathrm{C}$ in $5 \%$ carbon dioxide, then fixed in methanol at $-20^{\circ} \mathrm{C}$ or in $4 \%$ paraformaldehyde for $30 \mathrm{~min}$, and washed in PBS before removing the membranes from the plastic inserts.

Isolation of olfactory ensheathing cells. Neonatal Sprague Dawley rats [postnatal day 7 (P7)-P8] were killed by decapitation, and the posterior half of the nasal septum with attached olfactory neuroepithelium was collected. The olfactory neuroepithelium was dissected from the nasal septum, and fine forceps were used to tease away large olfactory nerve fascicles from the lamina propria. These nerve fascicles were incubated in a mixture of trypsin-versene (Commonwealth Serum Laboratories), collagenase-dispase ( $1 \mathrm{mg} / \mathrm{ml}$; Sigma), and RQ1 DNase (10 U; Promega, Madison, WI) at $37^{\circ} \mathrm{C}$ for $15 \mathrm{~min}$. Enzymatic digestion was stopped by the addition of $500 \mu \mathrm{l}$ of medium containing $10 \%$ (v/v) fetal bovine serum (dialyzed to remove molecules below $1000 \mathrm{Da}$; Sigma), and the tissue was centrifuged at $1000 \mathrm{rpm}$ for $5 \mathrm{~min}$. The pellet of tissue was washed with warmed medium, recentrif uged for another $5 \mathrm{~min}$, and then resuspended in $1 \mathrm{ml}$ of culture medium. The olfactory nerve fascicles were mechanically dissociated by trituration until semidissociated and plated into two to three wells of a 24 -well plate (Nunc, Naperville, IL). The culture medium consisted of minimal essential D-valine medium (Sigma), 10\% (v/v) dialyzed fetal bovine serum, and gentamicin (50 $\mu \mathrm{g} / \mathrm{ml}$ ). OECs were maintained at $37^{\circ} \mathrm{C}$ in $5 \% \mathrm{CO}_{2}$ for $6 \mathrm{~d}$. OECs were then subcultured as a dissociated culture into four-well chamber slides as follows (Nunc). OECs were rinsed with PBS and then incubated in trypsin-versene for $5 \mathrm{~min}$. To inactivate the trypsin, $500 \mu \mathrm{l}$ of warmed medium was added to each well, and cells were dislodged by trituration. The cell suspension was pelleted $(1000 \mathrm{rpm})$ for $5 \mathrm{~min}$, washed with warmed medium, and recentrifuged. Cells were plated at 10,000 cells per well and cultured for another $6 \mathrm{~d}$. Cultures were fixed in methanol for 20 min at $-20^{\circ} \mathrm{C}$, washed with PBS, and stored at $4^{\circ} \mathrm{C}$.

Olfactory ensheathing cells on ECM-coated coverslips. Round glass coverslips $(12 \mathrm{~mm})$ were cleaned overnight in concentrated nitric acid, washed thoroughly with distilled water, and air dried. Coverslips were then immersed in $70 \%$ ethanol (v/v) and flame sterilized. Coverslips were coated with poly-L-lysine $(1 \mu \mathrm{g} / \mathrm{ml})$ for $2 \mathrm{hr}$, washed with PBS, and then coated with various ECM molecules for another $2 \mathrm{hr}$. The different substrata used were as follows: chondroitin sulfate proteoglycan $(0.1,1$, and $10 \mu \mathrm{g} / \mathrm{ml})$, heparan sulfate proteoglycan $(0.1,1$, and $10 \mu \mathrm{g} / \mathrm{ml})$, EHS-Matrigel $(15.4,154$, and $1540 \mu \mathrm{g} / \mathrm{ml})$, and EHS-laminin (1, 10, and $100 \mu \mathrm{g} / \mathrm{ml}$ ). OECs were subcultured as described above and plated on to the coated glass coverslips at a density of 3000 cells per coverslip. Twenty-four hours after plating, coverslips were washed with fresh medium and fixed in methanol for $20 \mathrm{~min}$ at $-20^{\circ} \mathrm{C}$ and washed in PBS before immunostaining with the rabbit polyclonal p75 antiserum (Promega).

Neural cell adhesion molecule immunostaining of explant cultures. Explant cultures that had been fixed in methanol were washed with Trisbuffered saline (TBS), $\mathrm{pH} 7.4$, blocked with $2 \%$ bovine serum albumin (BSA) (Sigma) in TBS, and incubated overnight at $4^{\circ} \mathrm{C}$ with a rabbit polyclonal neural cell adhesion molecule (N-CAM) antiserum (1:500) (Akeson et al., 1988). Explants were washed three times with TBS, incubated with a goat anti-rabbit antiserum conjugated to fluorescein isothiocyanate (FITC) (1:50; Sigma) for $1 \mathrm{hr}$, and rewashed. Membranes were then mounted on glass slides in a glycerol-based mounting medium under coverslips. Immunostaining was visualized using a Bio-Rad (Hercules, CA) MRC-1024 confocal scanning laser microscope. Serial optical sections of each explant were collected and then merged digitally. Montages of individual explants were constructed using Adobe Photoshop 5.0 software (Adobe Systems, San Jose, CA).

Double-label immunostaining of explant cultures. Explants previously fixed in $4 \%$ paraformaldehyde were washed with TBS and $0.3 \%$ Triton $\mathrm{X}-100, \mathrm{pH} 7.4$, and blocked with $2 \%$ BSA in TBS and $0.3 \%$ Triton X-100. Explants were incubated overnight at $4^{\circ} \mathrm{C}$ with mouse ascites against $\beta$-tubulin III (1:200; Sigma), washed with TBS and $0.3 \%$ Triton X-100, and then incubated overnight at $4^{\circ} \mathrm{C}$ with rabbit anti-human p75 immunoglobulins (1:200; Promega). Explants were then washed with TBS and $0.3 \%$ Triton $\mathrm{X}-100$, incubated for $1 \mathrm{hr}$ with horse anti-mouse immunoglobulins (1:200; Vector Laboratories, Burlingame, CA), and rewashed. Next, the tissue was incubated with tetramethyl rhodamine isothiocyanate-labeled extra avidin (1:50; Sigma) and FITC-labeled goat anti-rabbit immunoglobulins for $1 \mathrm{hr}$, washed thoroughly with TBS and $0.3 \%$ Triton X-100, mounted on glass slides, coverslipped with an aqueous mounting medium, and then visualized by confocal microscopy.

Immunostaining cultures of olfactory ensheathing cells. Primary cultures of olfactory ensheathing cells were washed with TBS and $0.3 \%$ Triton $\mathrm{X}-100$, followed by blocking in $2 \%$ BSA in TBS and $0.3 \%$ Triton X-100. After $1 \mathrm{hr}$, cells were incubated overnight at $4^{\circ} \mathrm{C}$ with polyclonal antibodies against GFAP (1:200; Dako, Carpinteria, CA), S-100 (1:1000; Dako Corporation), p75 (1:500), or with a monoclonal anti-p75 antibody (1:20; Boehringer Mannheim, Indianapolis, IN). Cells were washed with TBS and $0.3 \%$ Triton X-100 and incubated for $1 \mathrm{hr}$ with an appropriate biotinylated secondary antibody (1:200). The secondary antibodies used were horse anti-mouse immunoglobulins (Vector Laboratories) or goat anti-rabbit immunoglobulins (Vector Laboratories). The cultures were washed again and incubated for $1 \mathrm{hr}$ with the avidin-biotin horseradish peroxidase complex (Vectastain Elite ABC kit; Vector Laboratories). Next, the cultures were washed with TBS and $0.3 \%$ Triton X-100, rinsed in TBS, and then reacted with 3,3-diaminobenzidine $(0.5 \mathrm{mg} / \mathrm{ml})$ in the presence of $0.02 \% \mathrm{H}_{2} \mathrm{O}_{2}$. No nonspecific staining of olfactory ensheathing cells was observed in control incubations using either nonimmune sera or no primary antisera.

Characterization of olfactory ensheathing cells. The purity of dissociated olfactory ensheathing cell cultures was analyzed by determining the percentage of flat cells and spindle-like cells that expressed each glial marker. Cells were counted in three randomly selected fields from at least three different cell cultures. The number of cells that spread (cells with no birefringence under bright-field microscopy) on the ECM-coated coverslips was determined by counting the percentage of cells with spindle-like morphology (with processes) and flat ovoid morphology (without processes) in one field from each coverslip $(n=9)$. Counts were expressed as percentages and presented as the mean percentage of cells that spread or did not spread. Data were statistically analyzed by the Kruskal-Wallis and Mann-Whitney tests for nonparametric statistics. The total length of OEC processes was measured using the Image-Pro Plus image analysis computer package (Media Cybernetics, Silver Spring, MD). Digital images of 20 randomly selected OECs were recorded from three coverslips, and the length of every process was measured. Because OECs typically had one long process and one to two short processes, data were expressed as total length of processes per cell. Data were then averaged and statistically analyzed by one-way ANOVA and Tukey's multiple comparisons.

Neurite outgrowth from explant cultures. Neurite outgrowth from explants of olfactory neuroepithelium was quantified using the Image-Pro Plus computer package. Digital images of each explant $(n=82)$ were binarized, and the total surface area of neurites was measured. Axon area was measured from the edge of each explant and did not include any axons that had grown over the top of explants. Data were analyzed by one-way ANOVA and Tukey's multiple comparisons.

\section{RESULTS \\ Qualitative observations of explant cultures of olfactory neuroepithelium}

The expression pattern of various components of the ECM in the developing olfactory nerve pathway suggests that these molecules may modulate the growth and guidance of primary olfactory axons en route to the olfactory bulb. To investigate the interplay 

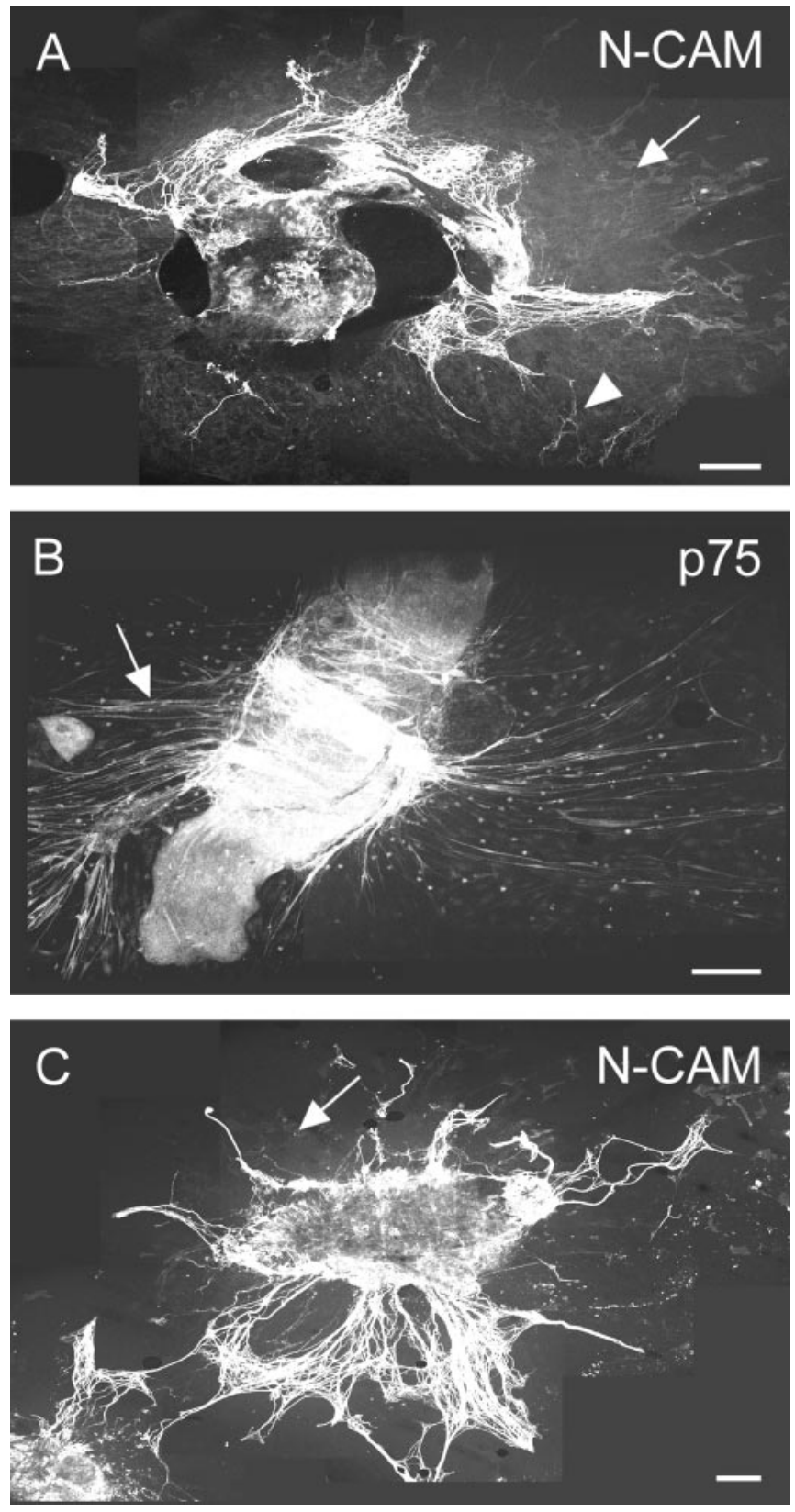

Figure 1. Explant cultures of E19.5 olfactory neuroepithelium grown on Matrigel $(154 \mu \mathrm{g} / \mathrm{ml})$. Explant in $A$ was grown in culture medium that did not contain any mitotic inhibitors. In $B$ and $C$, explants were grown in medium that contained cytosine arabinoside and fluorodeoxyuridine. $A$, $\mathrm{N}-\mathrm{CAM}$ immunostaining revealed bundles of olfactory neurites growing over the surface of OECs. OECs have formed a dense sheet of cells that weakly expressed N-CAM (arrow). Bundles of neurites defasciculate and grow between adjacent OECs (arrowhead). B, p75 is expressed by OECs (arrow), which have migrated from the explant. $C$, N-CAM immunostaining demonstrates large bundles of neurites growing over the surface of ensheathing cells. In these cultures, the extent of cell migration is markedly reduced (arrow). Scale bars, $200 \mu \mathrm{m}$.

between the ECM and migrating glia and olfactory neurites, explants of OE from E19.5 Sprague Dawley rats were plated onto Millicell-CM inserts coated with various substrates. When OE explants were cultured on a substrate of Matrigel for $5 \mathrm{~d}$, there was considerable cellular migration away from the explant (Fig. $1 A$ ). These cells formed a dense sheet between adjacent explants and were identified as OECs because they weakly expressed
N-CAM (Fig. 1A) and strongly expressed p75 (Fig. 1B). Growing over the surface of the sheet of ensheathing cells were numerous small fascicles of neurites, as well as N-CAM reactive olfactory neurons (Fig. $1 A, C$, arrows). The large amount of cell migration on Matrigel made it difficult to analyze and quantify the level of neurite outgrowth because the neurites were often in small bundles, which branched and defasciculated, interweaving between adjacent ensheathing cells (Fig. $1 A$, arrowhead). To decrease OEC proliferation, cytosine arabinoside and fluorodeoxyuridine were added to the culture medium. In the presence of these two mitotic inhibitors, the extent of OEC migration was considerably reduced and neurites grew in large bundles, rarely extending further than the edge of the OEC sheet (Fig. 1C).

\section{Neurite outgrowth is promoted when explants are plated on laminin and Matrigel}

We have proposed previously that expression of laminin and heparan sulfate proteoglycans in the olfactory nerve pathway provide a conducive substrate for axon growth, whereas expression of chondroitin sulfate proteoglycans surrounding the pathway act to restrict axon growth (Treloar et al., 1996). To examine the role of these ECM molecules, explants of olfactory neuroepithelium were cultured on substrates of PLL, Matrigel, laminin, HSPG, and CSPG. Olfactory neuroepithelium cultured on substrates of Matrigel and laminin had the highest level of neurite outgrowth, with neurites extending up to $1 \mathrm{~mm}$ from the explants, whereas explants plated on a substrate of PLL, HSPG, and CSPG had very little neurite outgrowth (Fig. 2). Interestingly, when explants were plated on PLL, HSPG, or CSPG, many neurites were confined to the surface or around the circumference of each explant (Fig. 2A-C). Furthermore, fewer ensheathing cells migrated away from explants cultured on these substrates (Fig. $2 B$, arrow). In contrast, both Matrigel and laminin promoted the migration of ensheathing cells (Fig. 2D,E, arrows). We also noticed that olfactory neurites preferentially grew across the surface of OECs rather than on the underlying ECM. To examine this in more detail, explants were double-labeled for the neural-specific type III isoform of $\beta$-tubulin, as well as for p75 (Fig. 3). Tubulin-positive neurites (Fig. 3A, red) clearly extended across the surface of ensheathing cells (Fig. $3 B$, green) and rarely contacted the underlying ECM. This is clearly apparent in merged images in which most axons were yellow-orange in appearance (Fig. 3C).

The extent of neurite outgrowth from the explants $(n=82)$ was determined by measuring the surface area of N-CAM-labeled processes in digital images. There was no significant difference between the mean area of neurite growth from explants cultured on either Matrigel or laminin (Fig. 4). However, the extent of neurite growth on Matrigel and laminin was significantly greater than on PLL, HSPG, or CSPG $(p<0.001)$. There were no significant differences in neurite outgrowth from explants cultured on PLL, HSPG, or CSPG (Fig. 4).

\section{Effect of ECM on olfactory ensheathing cells}

The above results suggested that neurite outgrowth was indirectly modulated by the ECM. It appeared that the ECM was modulating the neurite outgrowth-promoting properties of OECs. To examine the effect of ECM molecules on the spreading of these cells, we prepared dissociated cultures of OECs from the olfactory neuroepithelium. Previous studies have isolated OECs from the outer nerve fiber layer of the olfactory bulb (Chuah and Au, 1993; Doucette, 1993) or from whole olfactory neuroepithelium 

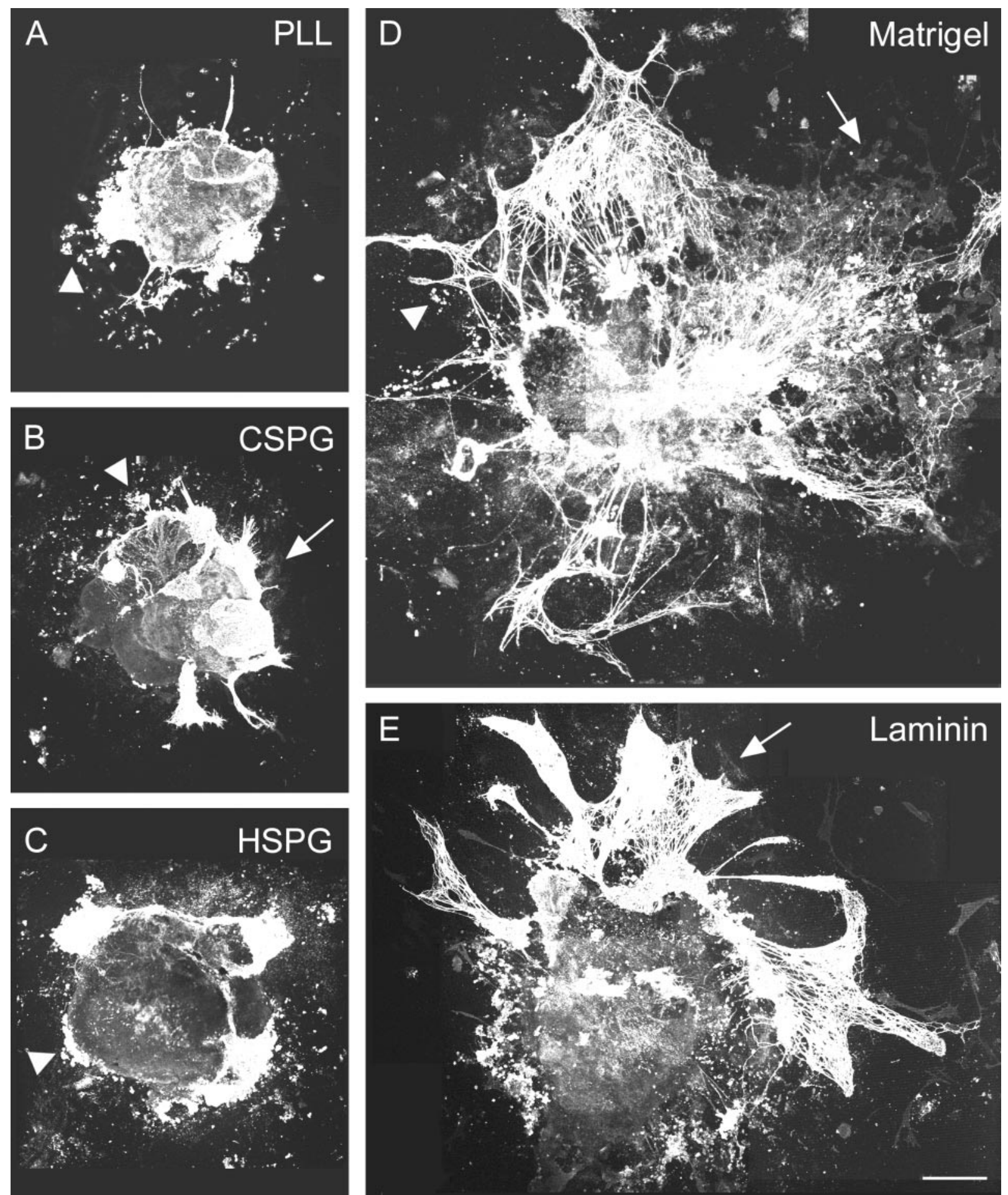

Figure 2. Explant cultures of E19.5 olfactory neuroepithelium plated on different substrates. Cultures were grown in the presence of cytosine arabinoside and fluorodeoxyuridine and were immunostained for N-CAM. When explants were plated on PLL $(A)(0.1 \mu \mathrm{g} / \mathrm{ml}), \mathrm{CSPG}(B)(1 \mu \mathrm{g} / \mathrm{ml})$, and HSPG $(C)$ $(1 \mu \mathrm{g} / \mathrm{ml})$, there was a low level of neurite outgrowth. Neurite outgrowth was confined to the upper surface and the edges of the explants. Large numbers of N-CAM-positive olfactory neurons (Calof and Chickaraishi, 1989) have migrated on these substrates (arrowheads). In contrast, there was minimal OEC migration on these substrates (arrow in B). When explants were plated on Matrigel $(D)(154 \mu \mathrm{g} / \mathrm{ml})$ and laminin $(E)(10 \mu \mathrm{g} / \mathrm{ml})$, there was extensive OEC migration (arrows) and neurite outgrowth. Neurites were in small bundles and extended up to $1 \mathrm{~mm}$ from the explants. Neurites only appeared to grow over underlying OECs. In addition, many olfactory neurons have migrated from the explants (arrowhead). Scale bar (in $E$ ): $A-E, 200 \mu \mathrm{m}$.

(Barber and Lindsay, 1982; Pixley, 1992). Both techniques produce OECs that are contaminated with other cells. In this study, we isolated OECs from olfactory nerve fascicles teased from the submucosa of the sheet of neuroepithelium lining the nasal sep- tum of P7-P8 Sprague Dawley rats. Semidissociated cultures were grown for $6 \mathrm{~d}$, triturated, replated into wells as dissociated cells, and then maintained in medium that reduced fibroblast proliferation. After $6 \mathrm{~d}$, ensheathing cell cultures were characterized by 

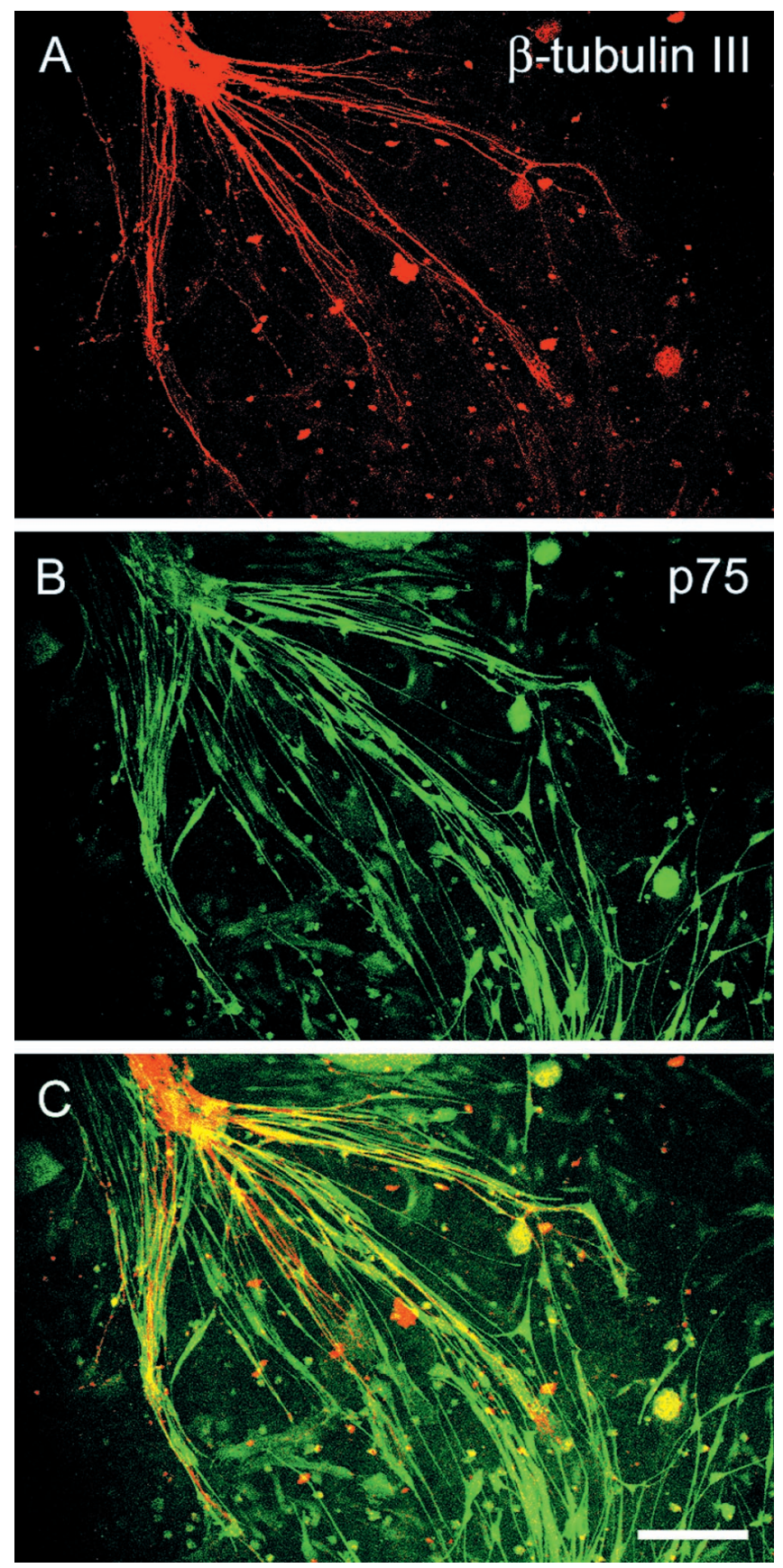

Figure 3. Double-label immunofluorescence of neurites extending from an explant plated on Matrigel $(154 \mu \mathrm{g} / \mathrm{ml})$. $A$, Immunostaining for $\beta$-tubulin III identifies small bundles of neurites extending from the explant. $B, \mathrm{p} 75$ labeling of the same field reveals that OECs are growing directly below the neurites. Ensheathing cells are spindle-shaped and have aligned in a longitudinal manner to form chains of cells. $C$, Double-label immunofluorescence reveals predominantly yellow-orange olfactory neurites, indicating that they preferentially grow over the surface of OECs rather than the underlying matrix. Scale bar (in $C$ ): $A-C, 100 \mu \mathrm{m}$.

morphology and immunostaining for p75, GFAP, and S-100 (Ramón-Cueto and Valverde, 1995). The majority of cells (96\%) were bipolar spindle-shaped (Fig. 5). The remaining cells (4\%) were highly spread and flat in appearance. These OEC cultures were very pure because almost all cells were positive for p75 (99.8\%), GFAP (99.0\%), and S-100 (98.0\%) (Fig. 5). A small number of flat cells did not express the highly specific OEC

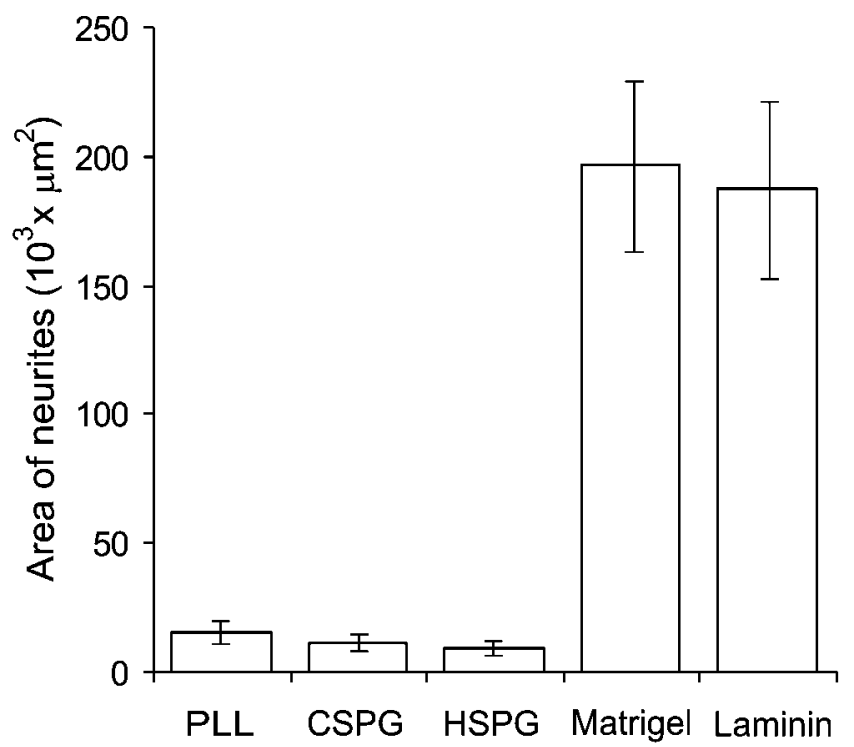

Figure 4. Quantification of the extent of neurite outgrowth from explant cultures on different ECM substrates. The surface area of neurites was measured from the edge of each explant $(n=82)$. Data were analyzed by one-way ANOVA and Tukey's multiple comparisons. The extent of neurite outgrowth on laminin and Matrigel was significantly greater than on PLL $(0.1 \mu \mathrm{g} / \mathrm{ml})$, CSPG $(1 \mu \mathrm{g} / \mathrm{ml})$, or HSPG $(1 \mu \mathrm{g} / \mathrm{ml})(p<0.001)$. There were no significant differences between the mean surface area of neurites on PLL, CSPG, or HSPG. The mean area of neurite outgrowth on Matrigel $(154 \mu \mathrm{g} / \mathrm{ml})$ and laminin $(10 \mu \mathrm{g} / \mathrm{ml})$ was also not significantly different. Error bars indicate the SEM. $n=82$ is the total number of explants analyzed with between 9 and 17 explants for each experiment.

marker p75 in these cultures (Fig. 5D, arrowhead). However, flattened triangular cells expressed S-100 (Fig. 5C, arrow), p75 (Fig. 5D, arrow), and GFAP (Fig. 5E, arrow).

Dissociated OECs were then replated onto coverslips coated with PLL $(1 \mu \mathrm{g} / \mathrm{ml})$, Matrigel $(154 \mu \mathrm{g} / \mathrm{ml})$, laminin $(10 \mu \mathrm{g} / \mathrm{ml})$, HSPG $(1 \mu \mathrm{g} / \mathrm{ml})$, or CSPG $(1 \mu \mathrm{g} / \mathrm{ml})$. After $24 \mathrm{hr}$, cultures were fixed and stained for p75. Many OECs that were plated on substrates of PLL, HSPG, or CSPG exhibited minimal spreading, and these cells were usually ovoid in shape (Fig. 6A-C, arrowheads). Only approximately half of the cells spread and obtained a spindle shape on these substrates (Figs. $6 A-C, 7 A$, arrows). In contrast, $\sim 95 \%$ of all OECs on Matrigel and laminin had spread out and differentiated into spindle-shaped cells (Figs. 6D, E, 7A, arrows). The percentage of spread OECs that adopted a spindle shape on Matrigel and laminin were significantly higher than those grown on PLL, CSPG, or HSPG $(p<0.0004)$ (Fig. $7 A)$. In addition, there were significantly more spindle-shaped OECs on HSPG than on PLL $(p<0.04)$. No significant differences were found between the percentage of cells that spread over HSPG compared with CSPG, and PLL compared with CSPG. Furthermore there were no significant differences in the percentage of OECs that spread at any concentration of CSPG, HSPG, or Matrigel (Fig. $7 A$ ). In contrast, altering the concentration of laminin affected the spreading of OECs. Approximately $95 \%$ of cells spread as spindle-shaped cells when 10 and $100 \mu \mathrm{g} / \mathrm{ml}$ of laminin were used, whereas on laminin at $1 \mu \mathrm{g} / \mathrm{ml}$, only $76.3 \%$ of cells exhibited this spindle shape (Fig. 7A). Although a different concentration of Matrigel substrates did not significantly affect the percentage of spread OECs, it did appear to change their morphology. OECs on high concentrations of Matrigel were more stellate, whereas on lower concentrations, the cells were more spindle-shaped. 

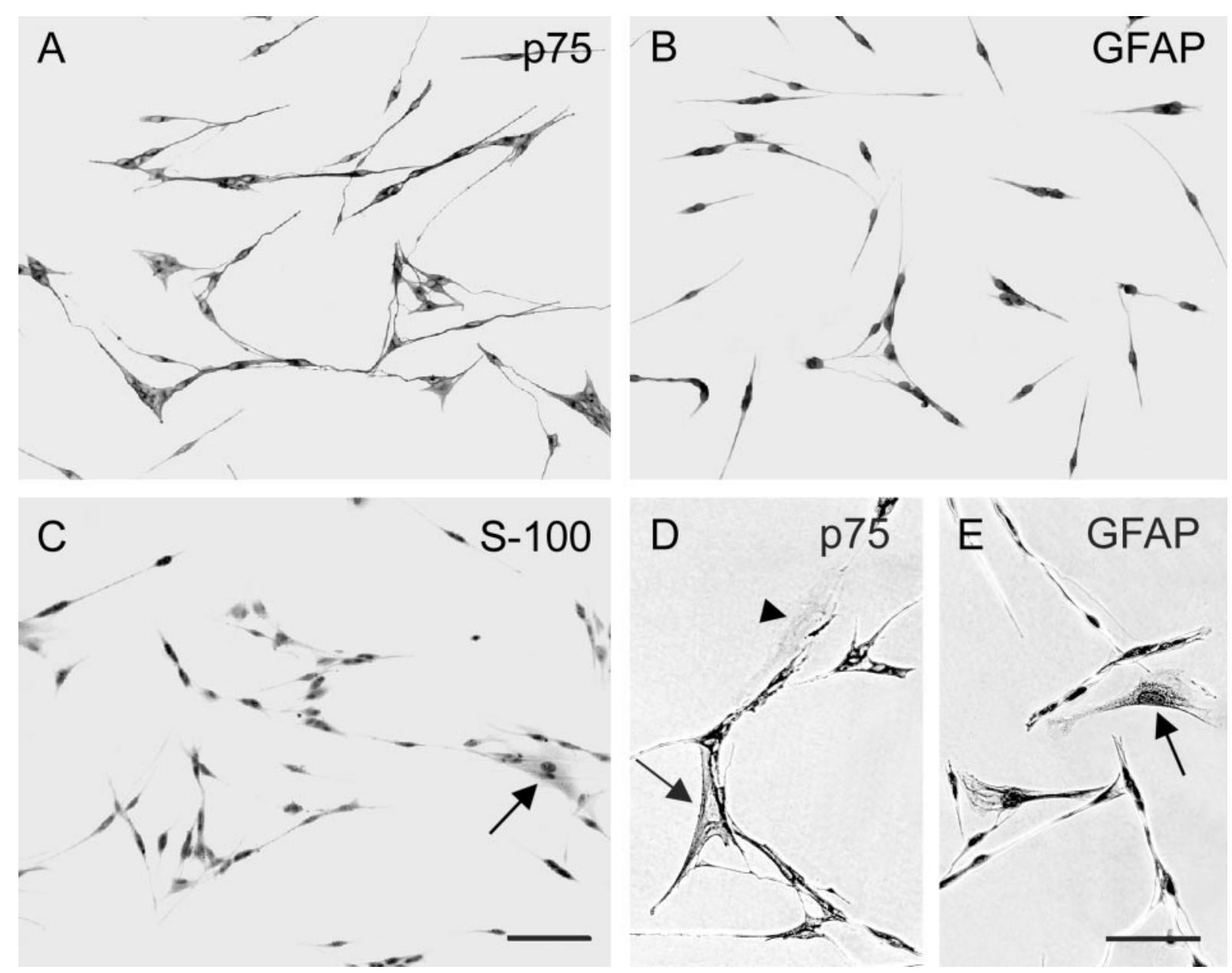

Figure 5. Dissociated cultures of OECs isolated from olfactory nerve fascicles. OECs were cultured for $12 \mathrm{~d}$ and then stained for p75 $(A)$, GFAP $(B)$, and S-100 $(C) . A-C$, Almost every cell expressed each of these markers. $D, E$, Phase contrast micrographs of p75 $(D)$ and GFAP $(E)$ demonstrate two cell morphologies. The majority of cells $(96 \%)$ have spindle-like morphology, whereas the other $4 \%$ of cells are flat and triangular in shape (arrows). In addition, there are a small number of unstained flattened cells in the cultures (arrowhead). Scale bars: (in $D$ ) $A-C, 200 \mu \mathrm{m}$; (in $E$ ) $D, E, 75 \mu \mathrm{m}$.

In addition to the low percentage of OECs that spread on PLL, HSPG, and CSPG, the length of spread processes were also considerably reduced. Spread cells on these substrates usually had one long process and one to two shorter processes that were straight and unbranched (Fig. 6A-C, arrows). In contrast, OECs on both Matrigel and laminin grew long thin processes, which were curved (Fig. $6 D, E$ ). The total length of processes per OEC was significantly longer on Matrigel and laminin compared with PLL, HSPG, or CSPG $(p<0.001)$ (Fig. 7B). There were no significant differences between the length of cell processes on PLL, CSPG, and HSPG or between Matrigel and laminin.

\section{DISCUSSION}

The growth cones of primary olfactory axons migrate toward the olfactory bulb over the surface of OECs, which are embedded in an ECM rich in laminin, HSPG, and collagen IV. These axons and cells are confined to a discrete pathway demarcated by the expression of CSPG and fibronectin in the surrounding mesenchyme (Treloar et al., 1996; Whitesides and LaMantia, 1996). We have shown here that Matrigel, an ECM secreted by EHS cells, regulates the shape of OECs and profoundly enhances the neurite outgrowth-promoting properties of these cells. Purified laminin, one of the constituents of Matrigel, also has a similar effect on OECs. In contrast, CSPGs and HSPGs do not facilitate spreading and appear to inhibit the neurite outgrowth-promoting properties of OECs.

\section{ECM modulates the growth of olfactory ensheathing cells}

We isolated ensheathing cells from fascicles of the olfactory nerve teased from the submucosa of the neuroepithelium lining the nasal septum. Almost every cell in these cultures expressed p75, GFAP, and S-100, which are phenotypic markers of OECs (Pixley, 1992; Ramón-Cueto and Valverde, 1995). Previous studies have isolated OECs from the outer nerve fiber layer of the olfactory bulb (Chuah and Au, 1993; Doucette, 1993; Goodman et al., 1993) or whole nasal epithelium (Barber and Lindsay, 1982; Pixley, 1992). However, the purity of OECs derived from these cultures is less than from the olfactory nerve. We have demonstrated, for the first time, that CSPG and HSPG do not support the spreading of OECs in vitro. Moreover, these molecules also appear to be nonpermissive substrates for migration of OECs from explant cultures of olfactory neuroepithelium. In contrast, Matrigel and laminin promoted cell spreading and migration of OECs. How does the ECM induce these changes in OECs? One possibility is that substrates of laminin and Matrigel provide an adhesive surface for attachment and spreading, whereas HSPG or CSPG may be unadhesive. However, the responsiveness of OECs 

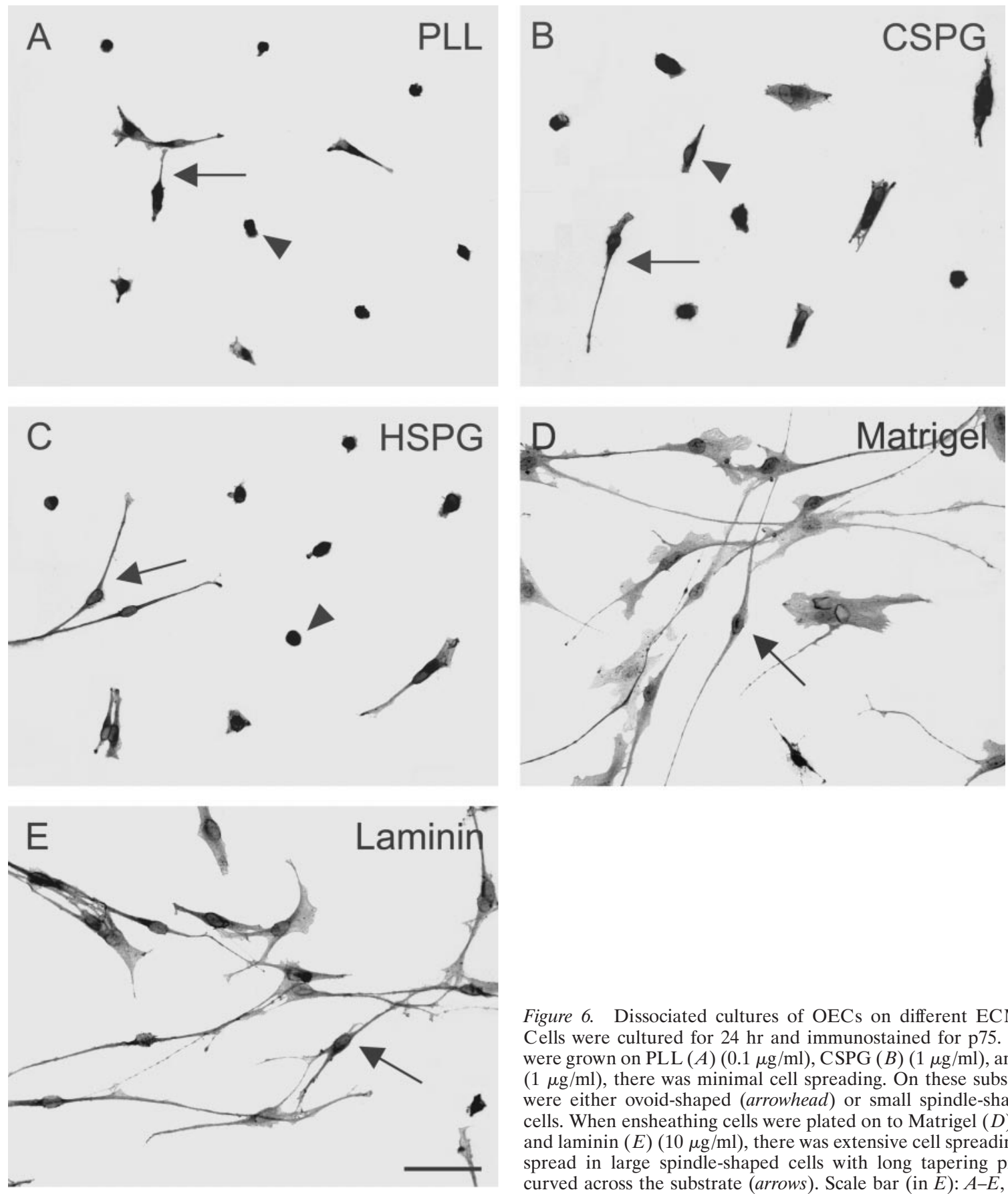

Figure 6. Dissociated cultures of OECs on different ECM substrates. Cells were cultured for $24 \mathrm{hr}$ and immunostained for $\mathrm{p} 75$. When OECs were grown on PLL $(A)(0.1 \mu \mathrm{g} / \mathrm{ml}), \operatorname{CSPG}(B)(1 \mu \mathrm{g} / \mathrm{ml})$, and HSPG $(C)$ $(1 \mu \mathrm{g} / \mathrm{ml})$, there was minimal cell spreading. On these substrates, OECs were either ovoid-shaped (arrowhead) or small spindle-shaped (arrows) cells. When ensheathing cells were plated on to Matrigel $(D)(154 \mu \mathrm{g} / \mathrm{ml})$ and laminin $(E)(10 \mu \mathrm{g} / \mathrm{ml})$, there was extensive cell spreading. Most cells spread in large spindle-shaped cells with long tapering processes that curved across the substrate (arrows). Scale bar (in $E$ ): $A-E, 100 \mu \mathrm{m}$.

to these molecules is unlikely the result of differential adhesiveness because there were no noticeable differences in the attachment of cells to any of the substrates. Moreover, PLL is highly adhesive and yet did not promote spreading to the same extent as either Matrigel or laminin. Thus, laminin and Matrigel appear to be specifically promoting the spreading, whereas HSPG and CSPG are either nonpermissive or inhibitory for spreading. Decreasing the concentration of laminin did not alter cell attachment; however, it did decrease the relative proportion of cells that spread as spindle-shaped cells. These results indicate that OECs respond dynamically to changes in the composition of the ECM. In contrast, altering the levels of HSPG and CSPG in the substrate had little effect on the spreading of OECs. Thus, HSPG and
CSPG appear to be nonpermissive rather than inhibitory for cell spreading.

Schwann cells, like OECs, are also influenced by the nature of the ECM. Schwann cells transfected with Syndecan-1, a cell surface HSPG, adopt a flat cuboid cell shape rather than the typical spindle-shaped morphology (Carey et al., 1994). Syndecan-1 was proposed to stimulate reorganization of the cytoskeleton by interacting with the actin filaments during cell spreading (Carey et al., 1994). HSPGs bind to several components of the ECM, including laminin (Carey et al., 1990), fibronectin (Saunders and Bernfield, 1988), and collagen (Koda et al., 1985), and stimulate adhesion and spreading of Schwann cells on laminin and fibronectin (Chernoff, 1988; Carey et al., 1990). We have 
A

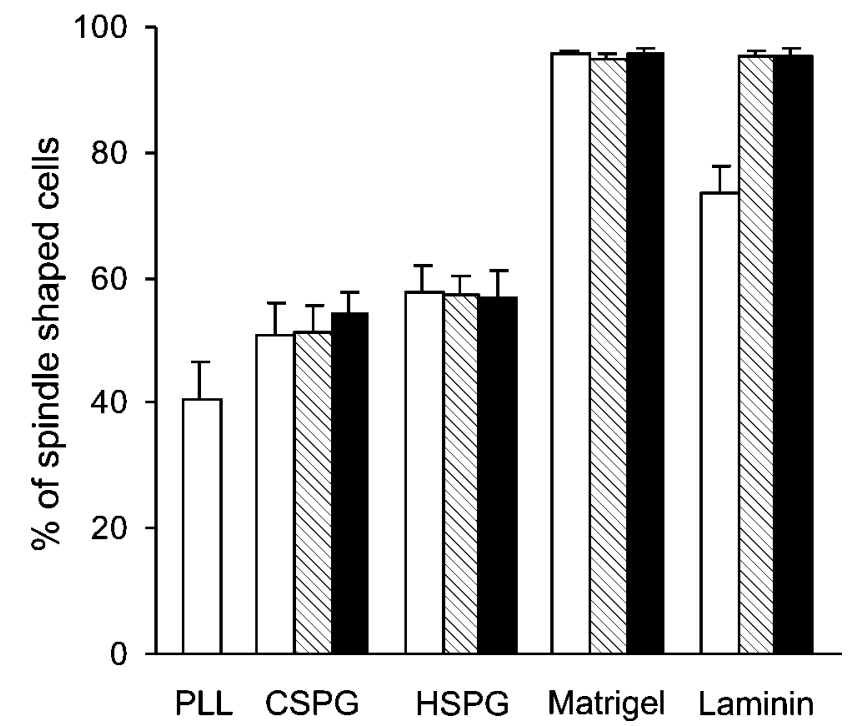

B

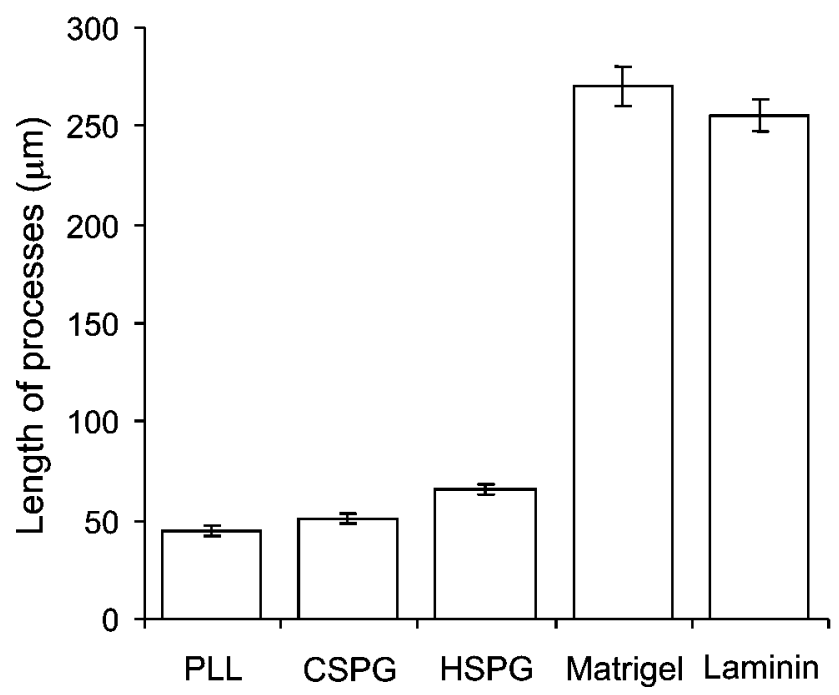

Figure 7. A, Quantification of cell spreading on different substrates. OECs were grown on coverslips coated with PLL $(0.1 \mu \mathrm{g} / \mathrm{ml})$, CSPG $(0.1$, 1 , and $10 \mu \mathrm{g} / \mathrm{ml})$, HSPG $(0.1,1$, and $10 \mu \mathrm{g} / \mathrm{ml})$, Matrigel $(15.4,154$, and $1540 \mu \mathrm{g} / \mathrm{ml})$, and laminin $(1,10$, and $100 \mu \mathrm{g} / \mathrm{ml})$. After $24 \mathrm{hr}$, cells were fixed and stained for $\mathrm{p} 75$, and the mean percentage of ensheathing cells that spread, as spindle-shaped cells, was determined. Data were collected from nine independent samples and statistically analyzed by the KruskalWallis and Mann-Whitney tests. The mean percentage of cells that spread on laminin and Matrigel was significantly different compared with PLL, CSPG, and HSPG $(p<0.0004)$. In addition, the mean percentage of cells that spread on HSPG was greater than on PLL $(p<0.04)$. There were no significant differences in the mean percentage of cells that spread on CSPG compared with HSPG, or PLL compared with CSPG. There were no significant differences in the percentage of ensheathing cells that spread on substrates of CSPG, HSPG, or Matrigel at any concentration used. However, the percentage of cells that spread on laminin at $1 \mu \mathrm{g} / \mathrm{ml}$ was significantly less than on laminin at concentrations of $10 \mu \mathrm{g} / \mathrm{ml}(p<$ $0.0008)$ and $100 \mu \mathrm{g} / \mathrm{ml}(p<0.0011)$. Error bars indicate SEM. $B$, Quantification of the length of processes from olfactory ensheathing cells cultured on different substrates. OECs were grown on PLL $(1 \mu \mathrm{g} / \mathrm{ml})$, Matrigel $(154 \mu \mathrm{g} / \mathrm{ml})$, laminin $(10 \mu \mathrm{g} / \mathrm{ml})$, HSPG $(1 \mu \mathrm{g} / \mathrm{ml})$, or CSPG $(1$ $\mu \mathrm{g} / \mathrm{ml}$ ) for $24 \mathrm{hr}$. The length of processes from 20 random cells from each substrate was then measured and expressed as total length of process per cell. This experiment was performed in triplicate. Data were then averaged and analyzed by one-way ANOVA and Tukey's multiple compari- demonstrated previously that interactions between FGF-1 and HSPG also promote the spreading of OECs in vitro (Key et al., 1996). Furthermore, interactions between FGF-2 and perlecan on the cell surface also promote the proliferation of OECs (Chuah and Teague, 1999). These data revealed that HSPG requires cofactors for functional activity, which is consistent with the results of the present study.

Cell spreading is an important prerequisite for cell migration and differentiation and is mediated by many cells via molecular interactions with the ECM, which lead to the formation of focal adhesions (Ridyard and Sanders, 1999). We demonstrated that, like cell spreading, the migration of OECs away from explants of olfactory neuroepithelium was substantially less on substrates of either CSPG or HSPG compared with Matrigel or laminin. Interestingly, CSPGs have been reported previously to restrict the migration of Schwann cells over CNS astrocytes (Ghirnikar and Eng, 1995). In contrast, laminin promotes Schwann cell migration in the peripheral nervous system (Bailey et al., 1993; Anton et al., 1994), a process that appears to require interactions between laminin and integrins (Milner et al., 1997). It is possible that similar mechanisms may be stimulating migration of OECs on laminin.

\section{ECM molecules and neurite outgrowth from olfactory neuroepithelium}

We demonstrated that substrates of Matrigel and laminin promoted the extension of neurites from explants of olfactory neuroepithelium when compared with PLL, CSPGs, and HSPGs. Laminin has been shown previously to directly promote the growth of olfactory neurites in vitro (Whitesides and LaMantia, 1996; Kafitz and Greer, 1997). However, we show here that olfactory axons prefer to grow on OECs when given the choice of either these cells or a substrate of laminin. These results are consistent with previous reports demonstrating that septal neurons and retinal explants also preferentially extend neurites on astrocytes or Schwann cell surfaces rather than on the underlying ECM (Pixley et al., 1987; Kleitman et al., 1988). Laminin seems to be indirectly affecting neurite outgrowth in our explant cultures by enhancing the neurite outgrowth-promoting properties of OECs. When the explants were plated onto PLL, CSPG, or HSPGs, OECs became a poorer substrate for neurite outgrowth. These results probably also reflect the nonpermissive nature of these molecules for spreading and migration of OECs away from explants. We have demonstrated previously that HSPGs regulate the neurite outgrowth-promoting activity of OECs. Although HSPG alone does not promote olfactory neurite growth, HSPG together with FGF-1 significantly promote olfactory neurite growth on OECs (Key et al., 1996). Thus, HSPG may not be inhibitory for neurite outgrowth but rather requires appropriate cofactors to become functionally active.

Previous studies have demonstrated that OECs promote axon growth. OECs promote the extension of olfactory neurites in vitro (Goodman et al., 1993; Chuah and Au, 1994; Key et al., 1996; Kafitz and Greer, 1998), and this growth is greater than that produced by laminin (Kafitz and Greer, 1999). The outgrowth-

\section{$\leftarrow$}

sons. The total length of processes per cell was significantly greater on Matrigel and laminin compared with PLL, CSPG, or HSPG $(p<0.001)$. There were no significant differences between the total length of processes of cells cultured on PLL, CSPG, or HSPG. The total length of processes of cells on Matrigel was not significantly different from those on laminin. Error bars indicate SEM. 
promoting activity of OECs is associated with both membranebound factors and diffusible factors (Chuah and Au, 1994; Kafitz and Greer, 1998, 1999) and is not limited to olfactory neurons. OECs are able to stimulate the growth of dorsal root ganglion axons into the spinal cord (Ramón-Cueto and Nieto-Sampedro, 1994), as well as the regeneration of central axons across spinal cord lesions (Li et al., 1997, 1998; Ramón-Cueto et al., 1998). Interestingly, our results suggest that presentation of OECs in an ECM rich in laminin and low in CSPG may enhance their regenerative capabilities. This is particularly important because glial scars that form at lesion sites are rich in CSPGs (McKeon et al., 1991).

\section{Model of axon growth between the nasal cavity and olfactory bulb}

We have shown previously that laminin and CSPGs have highly restricted patterns of expression in the frontonasal mesenchyme between the nasal cavity and olfactory bulb (Treloar et al., 1996). The clear dichotomy in response of OECs to these molecules, together with their expression patterns, suggests that they play an important role in the development of the olfactory nerve pathway. During development, OECs migrate toward the rostral telencephalon in response to soluble trophic factors released specifically by the presumptive olfactory bulb (Lui et al., 1995). These cells pioneer the olfactory nerve pathway and provide a substrate for growing primary olfactory axons (Tennent and Chuah, 1996). Interestingly, in the peripheral nervous system, Schwann cells also pioneer the developing axon pathways in the limb buds (Noakes et al., 1988). Our results revealed that olfactory neurites preferentially grow over ensheathing cells rather than the ECM. Neurite outgrowth was always confined to regions in which there were OECs, and maximal neurite outgrowth was observed on those ECM molecules that promoted the spreading and migration of OECs. We propose that OECs secrete laminin and HSPG, which act in an autocrine manner together with cofactors to cause these cells to become migratory and to adopt an axon growthpromoting phenotype. Expression of CSPGs in the surrounding mesenchyme, which are nonpermissive for migration, would confine growth of OECs to a specific pathway. Thus, the ECM indirectly provides growth and guidance cues for migrating olfactory axons during development. However, these ECM molecules are not alone in regulating the trajectory of these axons. A complex interplay between the ECM, cell adhesion molecules, and growth factors is probably required to establish the olfactory nerve pathway.

\section{REFERENCES}

Akeson RA, Wujek JR, Roe S, Warren SL, Small SJ (1988) Smooth muscle cells transiently express NCAM. Brain Res 464:107-120.

Anton ES, Sandrock Jr AW, Matthew WD (1994) Merosin promotes neurite growth and Schwann cell migration in vitro and nerve regeneration in vivo: evidence using an antibody to merosin, ARM-1. Dev Biol 164:133-146.

Bailey SB, Eichler ME, Villadiego A, Rich KM (1993) The influence of fibronectin and laminin during Schwann cell migration and peripheral nerve regeneration through silicon chambers. J Neurocytol 22:176-184.

Barber PC, Lindsay RM (1982) Schwann cells of the olfactory nerves contain glial fibrillary acidic protein and resemble astrocytes. Neuroscience 7:3077-3090.

Calof AL, Chikaraishi DM (1989) Analysis of neurogenesis in a mammalian neuroepithelium: proliferation and differentiation of an olfactory neuron precursor in vitro. Neuron 3:115-127.

Carey DJ, Crumbling DM, Stahl RC, Evans DM (1990) Association of cell surface heparan sulfate proteoglycans of Schwann cells with extracellular matrix proteins. J Biol Chem 265:20627-20633.

Carey DJ, Stahl RC, Cizmeci-Smith G, Asundi VK (1994) Syndecan-1 expressed in Schwann cells causes morphological transformation and cytoskeletal reorganization and associates with actin during cell spreading. J Cell Biol 124:161-170.

Chernoff EA (1988) The role of endogenous heparan sulfate proteoglycan in adhesion and neurite outgrowth from dorsal root ganglia. Tissue Cell 20:165-178.

Chuah MI, Au C (1991) Olfactory Schwann cells are derived from precursor cells in the olfactory epithelium. J Neurosci Res 29:172-180.

Chuah MI, Au C (1993) Cultures of ensheathing cells from neonatal rat olfactory bulbs. Brain Res 601:213-220.

Chuah MI, Au C (1994) Olfactory cell cultures on ensheathing cell monolayers. Chem Senses 19:25-34.

Chuah MI, Teague R (1999) Basic fibroblast growth factor in the primary olfactory pathway: mitogenic effect on ensheathing cells. Neuroscience 88:1043-1050.

DeLorenzo AJ (1957) Electron microscope observations of the olfactory mucosa and olfactory nerve. J Biophys Biochem Cytol 3:839-850.

Devon R, Doucette R (1992) Olfactory ensheathing cells myelinate dorsal root ganglion neurites. Brain Res 589:175-179.

Doucette R (1984) The glial cells in the nerve fiber layer of the rat olfactory bulb. Anat Rec 210:385-391.

Doucette R (1990) Glial influences on axonal growth in the primary olfactory system. Glia 3:433-449.

Doucette R (1993) Glial progenitor cells of the nerve fiber layer of the olfactory bulb: effect of astrocyte growth media. J Neurosci Res 35:274-287.

Doucette R (1996) Immunohistochemical localization of laminin, fibronectin and collagen type IV in the nerve fiber layer of the olfactory bulb. Int J Dev Neurosci 14:945-959.

Ghirnikar RS, Eng LF (1995) Chondroitin sulfate proteoglycan staining in astrocyte-Schwann cell co-cultures. Glia 14:145-152.

Goodman MN, Silver J, Jacobberger JW (1993) Establishment and neurite outgrowth properties of neonatal and adult rat olfactory bulb glial cell lines. Brain Res 619:199-213.

Kafitz KW, Greer CA (1997) Role of laminin in axonal extension from olfactory receptor cells. J Neurobiol 32:298-310.

Kafitz KW, Greer CA (1998) The influence of ensheathing cells on olfactory receptor cell neurite outgrowth in vitro. Ann NY Acad Sci 855:266-269

Kafitz KW, Greer CA (1999) Olfactory ensheathing cells promote neurite extension from embryonic olfactory receptor cells in vitro. Glia 25:99-110.

Katoh-Semba R, Matsuda M, Kato K, Oohira A (1994) Chondroitin sulphate proteoglycans in the rat brain: candidates for axon barriers of sensory neurons and the possible modification by laminin of their actions. Eur J Neurosci 7:613-621.

Key B, Treloar HB, Wangerek L, Ford MD, Nurcombe V (1996) Expression and localization of FGF-1 in the developing rat olfactory system. J Comp Neurol 366:197-206.

Kleitman N, Wood P, Johnson MI, Bunge RP (1988) Schwann cell surfaces but not extracellular matrix organized by Schwann cells support neurite outgrowth from embryonic rat retina. J Neurosci 8:653-663.

Koda JE, Rapraeger A, Bernfield M (1985) Heparan sulfate proteoglycans from mouse mammary epithelial cells. Cell surface proteoglycan as a receptor for interstitial collagens. J Biol Chem 260:8157-8162.

Li Y, Field PM, Raisman G (1997) Repair of adult rat corticospinal tract by transplants of olfactory ensheathing cells. Science 277:2000-2002.

Li Y, Field PM, Raisman G (1998) Regeneration of adult rat corticospinal axons induced by transplanted olfactory ensheathing cells. J Neurosci 18:10514-10524.

Liu KL, Chuah MI, Lee KK (1995) Soluble factors from the olfactory bulb attract olfactory Schwann cells. J Neurosci 15:990-1000.

McKeon RJ, Schreiber RC, Rudge JS, Silver J (1991) Reduction of neurite outgrowth in a model of glial scarring following CNS injury is correlated with the expression of inhibitory molecules on reactive astrocytes. J Neurosci 11:3398-3411.

Milner R, Wilby M, Nishimura S, Boylen K, Edwards G, Fawcett J, Streuli C, Pytela R, ffrench-Constant C (1997) Division of labour of Schwann cell integrins during migration on peripheral nerve extracellular matrix ligands. Dev Biol 185:215-228.

Noakes PG, Bennett MR, Stratford J (1988) Migration of Schwann cells and axons into developing chick forelimb muscles following removal of either the neural tube or the neural crest. J Comp Neurol 277:214-233. 
Pixley SK (1992) The olfactory nerve contains two populations of glia, identified both in vivo and in vitro. Glia 5:269-284.

Pixley SK, Nieto-Sampedro M, Cotman CW (1987) Preferential adhesion of brain astrocytes to laminin and central neurites to astrocytes. J Neurosci Res 18:402-406.

Ramón-Cueto A, Nieto-Sampedro M (1992) Glial cells from adult rat olfactory bulb: immunocytochemical properties of pure cultures of ensheathing cells. Neuroscience 47:213-220.

Ramón-Cueto A, Nieto-Sampedro M (1994) Regeneration into the spinal cord of transected dorsal root axons is promoted by ensheathing glia transplants. Exp Neurol 127:232-244.

Ramón-Cueto A, Valverde F (1995) Olfactory bulb ensheathing glia: a unique cell type with axonal growth-promoting properties. Glia 14:163-173.

Ramón-Cueto A, Plant GW, Avila J, Bunge MB (1998) Long-distance axonal regeneration in the transected adult rat spinal cord is promoted by olfactory ensheathing glia transplants. J Neurosci 18:3803-3815.

Ridyard MS, Sanders EJ (1999) Potential roles for focal adhesion kinase in development. Anat Embryol (Berl) 199:1-17.

Saunders S, Bernfield M (1988) Cell surface proteoglycan binds mouse mammary epithelial cells to fibronectin and behaves as a receptor for interstitial matrix. J Cell Biol 106:423-430.

Tennent R, Chuah MI (1996) Ultrastructural study of ensheathing cells in early development of olfactory axons. Dev Brain Res 95:135-139.

Treloar HB, Nurcombe V, Key B (1996) Expression of extracellular matrix molecules in the embryonic rat olfactory pathway. J Neurobiol 31:41-55.

Whitesides III JG, LaMantia AS (1996) Differential adhesion and the initial assembly of the mammalian olfactory nerve. J Comp Neurol 373:240-254. 